\title{
The dopamine D1 receptor gene is associated with negative schizotypy in a non-clinical sample.
}

\section{To The Editors:}

The concept of a psychosis continuum, based on the notion that individual psychotic symptoms and subclinical schizotypic traits occur in the general population, has gained much support over recent years. Accumulating evidence suggests considerable overlap between schizotypy and schizophrenia, not simply at a phenomenological level but also in relation to cognitive, perceptual, neuropathological, psychopharmacological, environmental and genetic risk factors (Ettinger et al., 2014). In relation to psychopharmacological factors, the dopamine hypothesis of schizophrenia is longstanding and broadly suggests that positive symptoms may be partially attributable to changes in dopamine synthesis, release and a failure to regulate dopamine D2-like receptors, predominantly at a subcortical level, whereas, deficient dopaminergic transmission at D1 receptors in the prefrontal cortex (PFC) may contribute to the cognitive and negative symptoms of schizophrenia (Laruelle, 2014). The role of dopamine dysregulation in schizotypy is less established; however, there are pharmacological studies supporting a role for dopamine in schizotypy, as well as preliminary research linking polymorphisms in dopamine regulating genes to schizotypy (Colizzi et al., 2015; Grant et al., 2013; Taurisano et al., 2014). The DRD1 gene encodes the D1 subtype of dopamine receptor. One single nucleotide polymorphism (SNP), rs4532 (or DdeI), results in an A to G transition in the $5^{\prime}$ untranslated region (5'UTR) at bp-48 (A-48G) and has been described as a genuine, disease modifying risk allele in schizophrenia in a systematic meta-analysis (Allen et al., 2008).

We explored the association between rs4532 DRD1 and schizotypy in a non-clinical sample of 127 healthy adults ( $50 \%$ male; age $M=33.24$ years, $S D=13.22)$. Ethnicity was assessed using self-report (81\% Caucasian; 12.5\% Asian; 8\% Hispanic; 4.7\% 'Other'). Exclusion criteria were current Axis I mental illness, neurological disorder or other serious medical condition, brain injury, current substance dependence or a first degree biological relative with psychosis. To assess schizotypy, participants completed the 104 item self-report OxfordLiverpool Inventory of Feelings and Experiences (O-LIFE) scale (Mason and Claridge, 2006). Subscale scores reflecting factors of schizophrenia were calculated: Unusual Experiences or positive schizotypy; Introvertive Anhedonia or negative schizotypy, and Cognitive 
Disorganisation.. Participants also donated a blood sample for genetic analyses. Informed written consent was provided in accordance with The Alfred Hospital ethics committee requirements.

DNA was extracted from whole blood using QIAamp DNA Blood Mini Kit (QIAGEN, Hilden, Germany) and SNP assays were designed using the Sequenom Assay Design Suite 1.0 software (Sequenom, San Diego CA). Genotyping for rs4532 (CC, CT, TT) was carried out using the MassARRAY system (Sequenom, San Diego CA) as per the manufacturer's standard protocols. The MassArray platform relies on a primer extension reaction in combination with a mix of mass-tagged dideoxy-nucleotides (iPlex Gold chemistry) to generate a pool of oligo products that are analysed by chip-based matrix-assisted laser desorption/ionization time-of-flight mass spectrometry (MALDI-TOF). Adherence to HardyWeinberg equilibrium and allele frequency were assessed to ensure validity of the results.

The relationships between genotype and demographic characteristics were analysed using one-way analysis of variance (ANOVA) or chi square tests as appropriate. O-LIFE subscale scores were entered into three separate ANCOVAs with DRD1 genotypes (CC, CT, TT) entered as an independent variable, and age, ethnicity and sex entered as covariates. Following bonferroni corrections, a p-value of 0.02 was considered significant.

The overall mean scores for the schizotypy subscales were: Unusual Experiences $M=5.83$, $S D=5.10$, range: 0 - 25; Introvertive Anhedonia $\mathrm{M}=5.07, S D=4.47$, range: 0 - 20; Cognitive Disorganisation $\mathrm{M}=7.98, S D=5.39$, range: 0-20. Genotype-specific demographics indicated no significant group differences with respect to age $F(2,124)=0.63, p=0.53$, ethnicity $\chi^{2}$ (6 $N=127)=5.68, p=.46$ or $\operatorname{sex} \chi^{2}(2 N=127)=1.38, p=.50$. ANCOVAs revealed a significant main effect for $D R D 1 \mathrm{rs} 4532$ on Introvertive Anhedonia $F(2,121)=4.81, p=.010, p \eta^{2}=.074$, but not Cognitive Disorganisation $F(2,121)=1.84, p=.16, p \eta^{2}=.029$ or Unusual Experiences $F(2,121)=0.32, p=.73, p \eta^{2}=.005$.

This is the first report of an association between the gene that encodes the dopamine D1 receptor and schizotypy. Importantly, this study revealed that the factors of schizotypy resembling the negative symptoms of schizophrenia are associated with the minor rs $4532 / \mathrm{C}$ allele of $4532 \mathrm{SNP}$ on the $D R D 1$ gene. This finding extends previous research implicating the DRDI SNP as a risk gene for schizophrenia (Allen et al., 2008; although we note that a 
recent multi-stage genome wide association study that identified 108 schizophrenia associated genetic loci did not implicate DRD1, Schizophrenia Working Group of the Psychiatric Genomics, 2014), as well as research suggesting a role for dopamine in negative symptom schizotypy (Ettinger et al., 2013; Grant et al., 2013).

Previous research suggests that the major allele of $D R D 1$ (i.e. the $\mathrm{T}$ allele) increases gene expression, potentially via linkage disequilibrium with rs686 located in the 3'UTR (Huang et al., 2008). The minor rs $4532 / \mathrm{C}$ allele (potentially associated with a decrease in D1 expression) has been associated with a large set of phenotypes including addictive behaviours (e.g. nicotine addiction; Huang et al., 2008). In relation to schizophrenia, the minor rs4532/C allele has been associated with treatment resistance (e.g.Ota et al., 2012).

Our findings that the minor rs $4532 / \mathrm{C}$ allele of the $D R D 1$ gene is associated with increased scores on schizotypy factors that resemble negative symptoms in clinically diagnosable schizophrenia suggest that schizotypy is potentially linked to reduced D1 expression. This fits well with theories that dysregulation in dopaminergic transmission at D1 receptors in the PFC is associated with the negative symptoms of schizophrenia (Laruelle, 2014). For example, a D1-antagonist ( $\mathrm{SCH}$ 39166) has been shown to significantly reduce negative symptoms without impacting positive symptoms in schizophrenia (Den Boer et al., 1995). Furthermore, a positron emission tomography study of D1 receptors in drug-free patients with schizophrenia revealed a reduction in D1 receptor binding in the PFC that correlated with negative symptom severity and cognitive deficits (Okubo et al., 1997). Hence, there is some evidence linking dopaminergic transmission at D1 receptors to the negative symptoms associated with schizophrenia.

These results should be interpreted with caution as they are limited by the small sample size, as well as the use of a single SNP. Furthermore, our mean schizotypy scores for the Unusual Experience and Cognitive Disorganisation subscales were lower than other published norms (e.g. Mason and Claridge, 2006). Nevertheless, these positive, albeit preliminary findings encourage further studies with larger sample sizes (with a broader range of schizotypy scores) to explore schizotypy-relevant correlates of the $D R D 1$ gene with greater coverage.

To our knowledge, this is the first study demonstrating an association between a polymorphism in the DRD1 gene and schizotypy. The findings from this study add to the 
growing body of evidence that schizotypy and schizophrenia share a common biological basis related to genetic susceptibility, as well as shared pathological processes in the sense of dysregulation of dopamine-functioning. 


\section{References}

Allen, N.C., Bagade, S., McQueen, M.B., Ioannidis, J.P., Kavvoura, F.K., Khoury, M.J., Tanzi, R.E., Bertram, L., 2008. Systematic meta-analyses and field synopsis of genetic association studies in schizophrenia: the SzGene database. Nature genetics 40, 827-834.

Colizzi, M., Iyegbe, C., Powell, J., Ursini, G., Porcelli, A., Bonvino, A., Taurisano, P., Romano, R., Masellis, R., Blasi, G., Morgan, C., Aitchison, K., Mondelli, V., Luzi, S., Kolliakou, A., David, A., Murray, R.M., Bertolino, A., Di Forti, M., 2015. Interaction Between Functional Genetic Variation of DRD2 and Cannabis Use on Risk of Psychosis. Schizophrenia bulletin 41, 1171-1182.

Den Boer, J.A., van Megen, H.J., Fleischhacker, W.W., Louwerens, J.W., Slaap, B.R., Westenberg, H.G., Burrows, G.D., Srivastava, O.N., 1995. Differential effects of the D1-DA receptor antagonist SCH39166 on positive and negative symptoms of schizophrenia. Psychopharmacology 121, 317-322.

Ettinger, U., Corr, P.J., Mofidi, A., Williams, S.C., Kumari, V., 2013. Dopaminergic basis of the psychosis-prone personality investigated with functional magnetic resonance imaging of procedural learning. Frontiers in human neuroscience 7, 130.

Ettinger, U., Meyhofer, I., Steffens, M., Wagner, M., Koutsouleris, N., 2014. Genetics, Cognition, and Neurobiology of Schizotypal Personality: A Review of the Overlap with Schizophrenia. Frontiers in psychiatry 5, 18.

Grant, P., Kuepper, Y., Mueller, E.A., Wielpuetz, C., Mason, O., Hennig, J., 2013. Dopaminergic foundations of schizotypy as measured by the German version of the Oxford-Liverpool Inventory of Feelings and Experiences (O-LIFE)-a suitable endophenotype of schizophrenia. Frontiers in human neuroscience 7, 1. 
Huang, W., Ma, J.Z., Payne, T.J., Beuten, J., Dupont, R.T., Li, M.D., 2008. Significant association of DRD1 with nicotine dependence. Human genetics 123, 133-140.

Laruelle, M., 2014. Schizophrenia: from dopaminergic to glutamatergic interventions. Current opinion in pharmacology 14, 97-102.

Mason, O., Claridge, G., 2006. The Oxford-Liverpool Inventory of Feelings and Experiences (O-LIFE): further description and extended norms. Schizophrenia research 82, 203211.

Okubo, Y., Suhara, T., Suzuki, K., Kobayashi, K., Inoue, O., Terasaki, O., Someya, Y., Sassa, T., Sudo, Y., Matsushima, E., Iyo, M., Tateno, Y., Toru, M., 1997. Decreased prefrontal dopamine D1 receptors in schizophrenia revealed by PET. Nature 385, 634636.

Ota, V.K., Spindola, L.N., Gadelha, A., dos Santos Filho, A.F., Santoro, M.L., Christofolini, D.M., Bellucco, F.T., Ribeiro-dos-Santos, A.K., Santos, S., Mari Jde, J., Melaragno, M.I., Bressan, R.A., Smith Mde, A., Belangero, S.I., 2012. DRD1 rs4532 polymorphism: a potential pharmacogenomic marker for treatment response to antipsychotic drugs. Schizophrenia research 142, 206-208.

Schizophrenia Working Group of the Psychiatric Genomics, 2014. Biological insights from 108 schizophrenia-associated genetic loci. Nature 511, 421-427.

Taurisano, P., Romano, R., Mancini, M., Giorgio, A.D., Antonucci, L.A., Fazio, L., Rampino, A., Quarto, T., Gelao, B., Porcelli, A., Papazacharias, A., Ursini, G., Caforio, G., Masellis, R., Niccoli-Asabella, A., Todarello, O., Popolizio, T., Rubini, G., Blasi, G., Bertolino, A., 2014. Prefronto-striatal physiology is associated with schizotypy and is modulated by a functional variant of DRD2. Frontiers in behavioral neuroscience 8 , 235. 


\section{Author List;}

Caroline Gurvich ${ }^{\mathbf{1}}$, Erica Neill ${ }^{1,2}$, Kiymet Bozaoglu ${ }^{3}$, Tamsyn E. Van Rheenen ${ }^{1,2,4}$, Eric $\operatorname{Tan}^{1}$, Stephanie Louise ${ }^{1,2}$ Susan L. Rossell ${ }^{1,2,5}$

${ }^{1}$ Monash Alfred Psychiatry research centre, Central Clinical School, Monash University and The Alfred, Melbourne, Australia, ${ }^{2}$ Brain and Psychological Sciences Research Centre (BPsyC), Faculty Health, Arts and Design, Swinburne University, Melbourne, Australia, ${ }^{3}$ Genomics and Systems Biology, Baker IDI Heart and Diabetes Institute, Australia. Swinburne University, Melbourne, Australia; ${ }^{4}$ Melbourne Neuropsychiatry Centre, Department of Psychiatry, University of Melbourne, Melbourne, Australia; ${ }^{5}$ St Vincent's Mental Health, Melbourne, Australia.

Correspondence: Dr Caroline Gurvich, Monash Alfred Psychiatry research centre (MAPrc), Monash University Central Clinical School and The Alfred Hospital, Level 4, 607 St Kilda Road, Melbourne, 3004, Australia. Tel: +61 39076 6585. Email: caroline.gurvich@monash.edu

\section{Financial Disclosures and Conflict of Interest}

This work was funded by a National Health and Medical Research Council project grant (NHMRC APP1060664) and funding received from Australian Rotary Health/Bipolar Expedition and the Helen McPherson Smith Trust-. CG was funded by a NHMRC early career fellowship (NHRMC: APP546262) and TV-R was funded by an Australian Postgraduate Award. All authors report no biomedical financial interests or potential conflicts of interest. 